\title{
Etat des recherches \\ sur le dromadaire à partir de l'analyse bibliométrique des publications. Cas particulier des recherches sur le chamelon
}

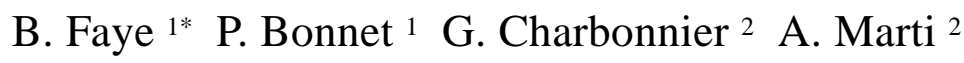

\begin{abstract}
Mots-clés
Dromadaire - Jeune animal - Documentation - Recherche - Coopération - Pays développé - Pays en développement.
\end{abstract}

\begin{abstract}
Résumé
Les activités de recherche sur le dromadaire ainsi que les principales collaborations entre les acteurs de ce secteur sont analysées en s'appuyant sur une étude bibliométrique concernant les 10 dernières années (publications dans les revues périodiques et les congrès). U ne attention particulière est apportée aux recherches sur le chamelon.
\end{abstract}

\section{INTRO DUCTION}

L'analyse des références obtenues à partir des bases de données bibliographiques est de plus en plus utilisée pour mieux comprendre la structure d'un domaine de recherche ou en faire un état des lieux. Cette méthode de mesure de la bibliographie est appelée bibliométrie. Elle permet, lorsque le nombre de références sur un sujet est de plusieurs centaines ou même de plusieurs milliers d'unités, d'extraire des informations difficiles à détecter par une simple lecture séquentielle. Elle s'appuie sur l'idée que la publication scientifique est un bon indicateur pour l'analyse des activités de la recherche.

L'analyse bibliométrique utilise les différents paramètres qui caractérisent une publication et qui sont donnés par la référence bibliographique. Traitables automatiquement, les données bibliométriques permettent de répondre aux questions du type « qui fait quoi ? où ? quand ? » et de mettre en relief les relations de coopération entre pays, organismes ou chercheurs.

Cette méthode appliquée aux références des publications faites sur le dromadaire depuis 1990 a permis d'établir une cartographie des travaux de recherche, d'identifier leur nationalité et de répertorier les acteurs majeurs, organismes et chercheurs, du domaine. Un regard particulier a été porté aux recherches réalisées sur le chamelon afin d'illustrer l'état de l'art dans le cadre de l'atelier international d'Ouarzazate. Cependant, la bibliométrie reste une méthode d'évaluation et les résultats ne reflètent que des tendances et non des preuves.

\footnotetext{
1. Cirad-emvt, Productions animales, TA30/A, 34398 Montpellier Cedex 5, France

2. Cirad-dist, T4383/5, 34398 Montpellier Cedex 5, France

* Auteur pour la correspondance et les tirés à part
}

\section{MATERIEL ET METHODES}

L'analyse présentée ici s'est limitée à la période 1990-1999. Le corpus documentaire a été constitué à partir des références bibliographiques rassemblées dans le cd-rom Le dromadaire (3), réactualisées pour l'année 1998 par l'interrogation des bases de données extérieures ( $\mathrm{Cab}$, Agris, Medline) et complétées par la saisie directe des communications aux colloques de 1998 à partir des actes non publiés. Seules les publications dans des revues périodiques et les communications à congrès ont été retenues.

Un important travail de normalisation du champ « organisme d'affiliation » des références a été effectué par retour au document primaire et par croisement automatique des données. En effet, en règle générale, les bases de données ne fournissent dans le champ « affiliation des auteurs » que celle du premier auteur, donnée insuffisante pour une étude des réseaux de coopération.

Un travail d'attribution de codes de classification à deux niveaux « domaine » et « thème » et d'homogénéisation a été réalisé avant traitement. Les références ont été traitées à l'aide d'un logiciel de traitement bibliométrique développé à l'université de Marseille (France).

\section{RESU LTATS}

\section{Vue d'ensemble de la recherche sur le dromadaire}

Le nombre de publications scientifiques retenues entre 1990 et 1999 s'élève à 1881 . Ce sont pour 66 p. 100 des articles de périodiques et pour le tiers restant des communications à des congrès.

La production annuelle d'articles de périodique depuis 1990 (figure 1) reste relativement stable même si les années 1995 et 1997 montrent une légère chute de productivité. Depuis 1990, 


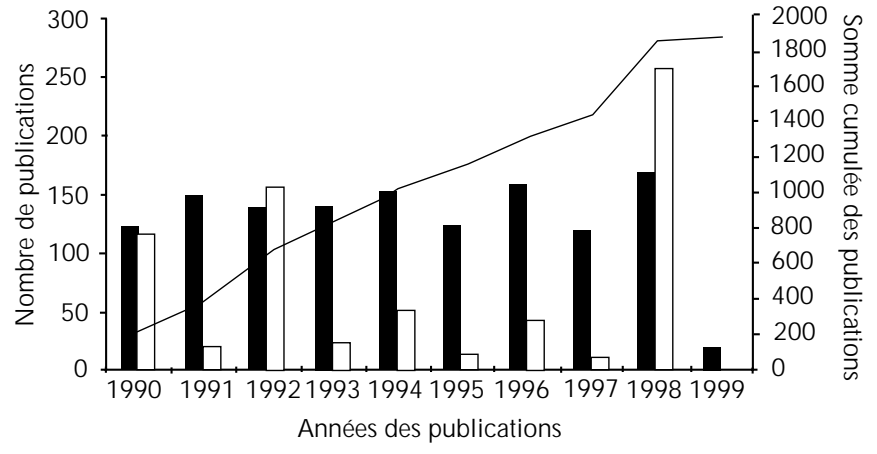

Articles de périodique $\square$ Communications à des congrès Somme cumulée des publications

Figure 1 : évolution du nombre des publications sur le dromadaire depuis 10 ans.

le nombre d'articles de périodiques publiés se situe entre 120 et 160 articles par an. Le dromadaire semble donc être un secteur de recherche qui a atteint une certaine maturité.

Le nombre de communications à des congrès (figure 1) est peu élevé en dehors des congrès spécialisés sur le dromadaire : en 1990 Tobrouk (Libye) et Paris (France), en 1992 Dubaï (Emirats arabes unis), en 1994 Nouakchott (Mauritanie), en 1996 Eilat (Israël), en 1998 Al Ain (Emirats arabes unis) et Bikaner (Inde). L'année 1999 n'est mentionnée qu'à titre indicatif, le nombre peu élevé de publications s'expliquant par les délais de mise à disposition de l'information dans les bases de données.

\section{Pays des acteurs impliqués dans la recherche sur le dromadaire}

Une liste de fréquence des publications faites au cours des huit dernières années a permis, à partir de la nationalité des auteurs, d'isoler 59 pays signataires ou cosignataires de publications sur le dromadaire.

Ils peuvent être classés en trois catégories (figure 2) :

- les pays incontournables, au nombre de quatre (44 p. 100 des publications totales), qui publient entre 20 et 42 articles par an ;

- les pays fortement impliqués, au nombre de 24, qui totalisent 51 p. 100 des publications et publient entre 2 et 12 articles chaque année ;

- les pays occasionnels, au nombre de 31, qui totalisent 5 p. 100 des publications et ne publient qu'un seul article par an.

Sur cette liste, on peut donc distinguer un groupe de 28 pays très actifs ou actifs. Il est à noter que ce groupe relève aussi bien de pays du Sud que du Nord.

\section{Pays du Sud}

Au nombre de 39, ils sont signataires ou cosignataires de 77 p. 100 des publications totales. Sept pays se remarquent par leur activité de publication (73 p. 100 des publications des pays du Sud) : l'Inde, l'Egypte, l'Arabie saoudite, les Emirats arabes, le Soudan, le Maroc et le Kenya. Il est à noter que le lien entre le nombre de publications pour un pays donné et l'importance économique du dromadaire dans ce même pays n'est pas automatique.

En ne s'intéressant qu'à des entités régionales constituées des pays originaires du dromadaire on constate que le Proche- et le MoyenOrient se situent en tête (462 publications), suivi du Maghreb (387) et du sous-Continent indien (382). Les pays de la Corne de l'Afrique qui possèdent la plus grande part du cheptel camelin mondial ne sont signataires que de 199 publications. Les autres pays de l'Afrique et de l'Asie viennent loin derrière (81 publications).

\section{Pays industrialisés}

Si 17 pays du Nord sont impliqués dans des activités de recherche sur le dromadaire, trois d'entre eux se distinguent nettement

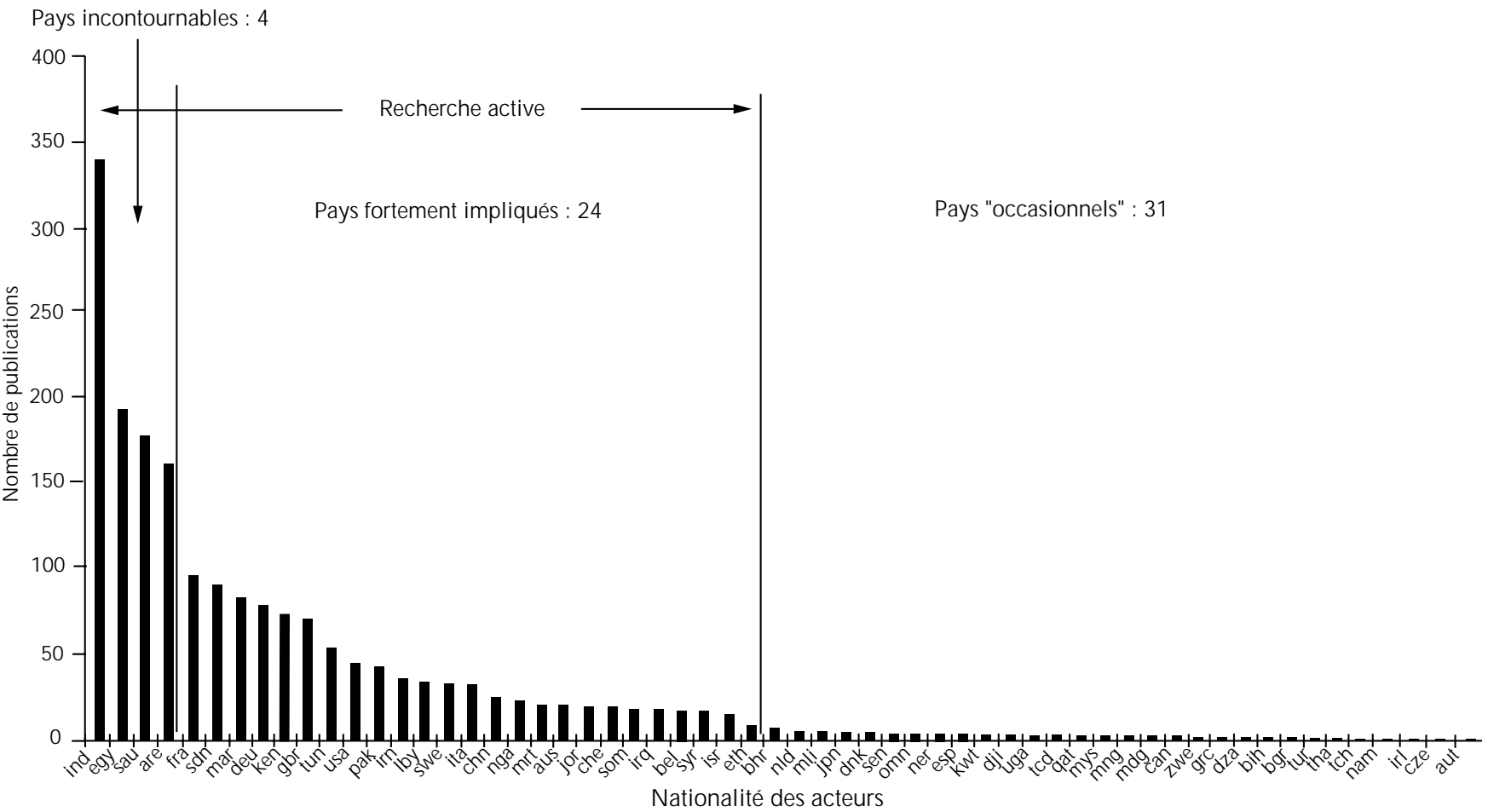

Figure 2 : répartition des pays par fréquence des publications sur le dromadaire (1990-1998). 
(figure 3) : la France, l'Allemagne et la Grande-Bretagne qui rassemblent 64 p. 100 des publications des pays européens. L'Australie, seul pays industrialisé à disposer d'un troupeau de dromadaires, est peu investi dans la recherche caméline.

Dans l'ensemble, le rang des pays leaders a été assez constant au cours des dix dernières années, témoignant d'une certaine stabilité des opérations de recherche. Dans les pays du Sud, les variations observées sont le plus souvent associées à la tenue des congrès. Parmi les pays européens, la France se situe le plus souvent au premier rang par son nombre de publications, quelle que soit l'année.

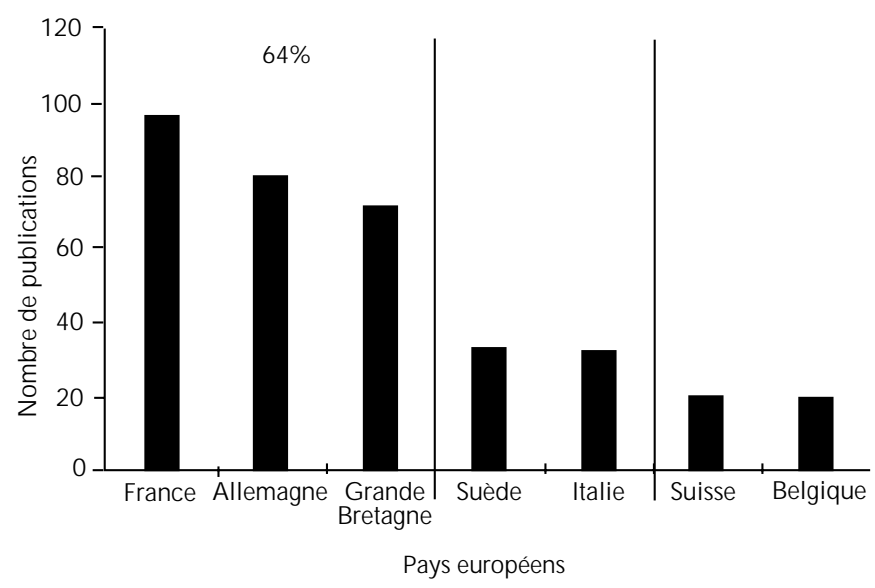

Figure 3 : classification des pays du Nord en fonction de leurs publications sur le dromadaire depuis 1990.

\section{Cartographie générale des domaines et des thèmes de recherche}

Les publications ont été indexées thématiquement à deux niveaux « domaine » et « thème » en se référant au plan de classement du Guide de l'élevage du dromadaire (2).

Cinq domaines ont été définis : 1) généralités (histoire, races, distribution, économie), 2) biologie (anatomie, physiologie, immunologie), 3) élevage (système de production, reproduction, croissance, lactation, alimentation, génétique, comportement), 4) productions (lait, viande, travail, cuirs et peaux), 5) maladies. Les références ne sont affectées que d'un seul code « domaine ». Globalement, sur huit ans, leur répartition souligne la prédominance des recherches liées aux maladies et à la biologie et ce, de façon stable dans le temps (tableau I). La part importante des publications en productions en 1994 est liée à la tenue du colloque de Nouakchott consacré à la production laitière chez la chamelle (1). Une analyse plus détaillée des thèmes à l'intérieur des domaines de recherche indiquent quelques tendances instructives.
En pathologie, les thèmes dominants sont les parasitoses internes et externes ( 19 p. 100 des références traitant des maladies), la pharmacologie (15 p. 100), les trypanosomes (15 p. 100) et les maladies bactériennes (14 p. 100). Les maladies virales et la traumatologie représentent chacune 10 p. 100 des publications sur les maladies. Pour l'ensemble de ces thèmes, l'activité de publication est régulière depuis 1990.

En biologie, le thème de la biochimie est nettement dominant (27 p. 100 des publications consacrées à la biologie). L'étude par année montre que le nombre de publications sur ce thème s'est accru depuis 1996. Les autres thèmes concernent par ordre d'importance, le système cardio-vasculaire (20 p. 100), le système urogénital (17 p. 100), l'endocrinologie (14 p. 100) et l'appareil digestif (13 p. 100). Les travaux sur l'immunologie et le système lymphatique n'apparaissent que depuis 1992.

En élevage, les deux thèmes de recherche dominants sont l'alimentation (34 p. 100) et la reproduction (31 p. 100). Le poids de ces deux thèmes peut varier au cours du temps. Les études sur la reproduction, par exemple, ont fortement dominé en 1990 (43 p. 100), du fait notamment de l'atelier de Paris (4), et de façon plus modérée en 1998 (28 p. 100). Le thème sur l'alimentation a largement été représenté en 1992 (49 p. 100) et en 1995 (44 p. 100). On peut également remarquer un engouement progressif pour ce dernier thème, notamment depuis 1998.

Pour les aspects relatifs à la production, le thème de la production laitière représente plus de la moitié (56 p. 100) des publications dans ce domaine.

\section{Répartition géographique des recherches}

La mise en relation de la nationalité des auteurs avec les domaines et les thèmes de recherche permet de mettre en évidence d'éventuelles spécificités ou spécialisation géographiques. Elle a été effectuée pour les pays du Sud et pour les pays du Nord.

\section{Pays du Sud}

Les résultats obtenus par regroupement géographique des pays (figure 4) montrent, à l'exception des pays d'Asie, que les maladies sont le domaine de recherche dominant. Elles représentent jusqu'à 64 p. 100 des publications des pays de la Corne de l'Afrique. Pour les pays du Maghreb et de la zone Proche- et Moyen-Orient, la part des recherches consacrée aux maladies et à la biologie est équivalente.

L'étude réalisée au niveau des pays (tableau II) situe de façon plus apparente les centres d'intérêt des pays leaders.

Trois autres pays présentant des spécificités intéressantes sont mentionnés :

- la Tunisie qui se caractérise par un nombre élevé de publications consacrées à l'élevage ;

\section{Tableau}

Part des différents domaines de recherche (en pourcentages)

\begin{tabular}{lrrrrrrrrr} 
Domaines & $\mathbf{1 9 9 0}$ & $\mathbf{1 9 9 1}$ & $\mathbf{1 9 9 2}$ & $\mathbf{1 9 9 3}$ & $\mathbf{1 9 9 4}$ & $\mathbf{1 9 9 5}$ & $\mathbf{1 9 9 6}$ & $\mathbf{1 9 9 7}$ & $\mathbf{1 9 9 8}$ \\
\hline Maladies & 27 & 39 & 41 & 45 & 41 & 44 & 43 & 42 & 43 \\
Biologie & 23 & 35 & 30 & 36 & 25 & 35 & 41 & 43 & 27 \\
Elevage & 28 & 13 & 17 & 9 & 9 & 16 & 8 & 11 & 19 \\
Productions & 16 & 8 & 8 & 6 & 23 & 4 & 4 & 2 & 8 \\
Généralités & 6 & 5 & 4 & 4 & 2 & 2 & 4 & 2 & 4
\end{tabular}




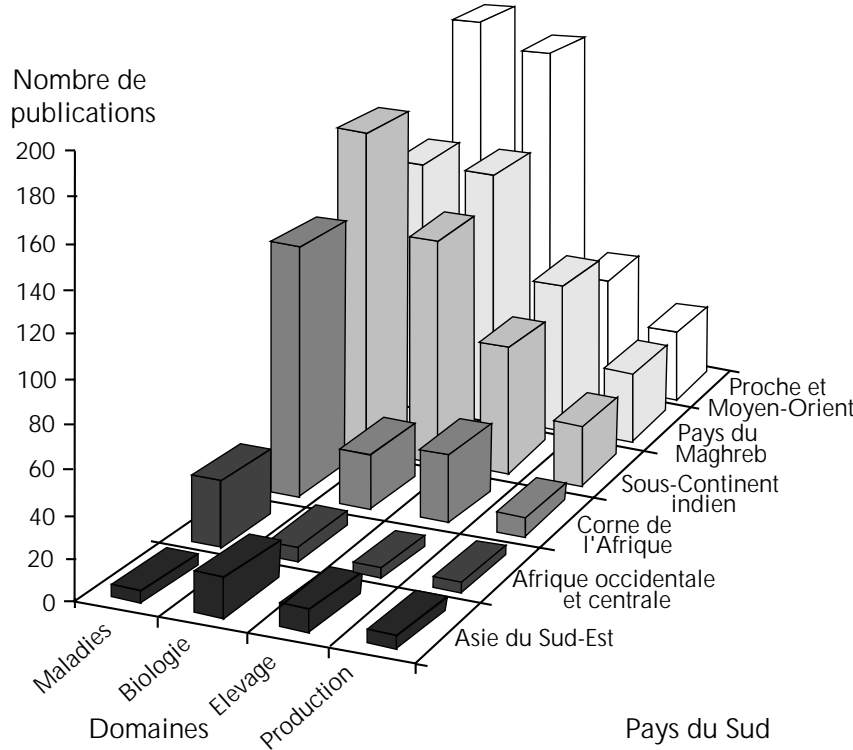

Figure 4 : répartition des publications sur le dromadaire par domaine pour les pays du Sud depuis 1990.

\section{Tableau II}

Répartition des publications par domaines de recherche depuis 1990 (en nombre de publications)

\begin{tabular}{lrrrc} 
Pays du Sud & Maladies & Biologie & Elevage & Production \\
\hline Inde & 139 & 103 & 58 & 28 \\
Egypte & 92 & 64 & 23 & 11 \\
Arabie saoudite & 64 & 80 & 13 & 21 \\
Emirats arabes unis & 77 & 43 & 29 & 7 \\
Soudan & 59 & 13 & 16 & 3 \\
Maroc & 19 & 46 & 14 & 3 \\
Kenya & 54 & 2 & 8 & 4 \\
Tunisie & 5 & 14 & 26 & 6 \\
Mauritanie & 16 & 1 & 1 & 3 \\
Iran & 7 & 25 & 4 & 1
\end{tabular}

- l'Iran qui publie régulièrement en biologie (depuis 1993 seulement);

- la Mauritanie qui publie sur les maladies du dromadaire (depuis 1994 seulement).

\section{Pays européens}

Les trois pays leaders, la France, l'Allemagne et la GrandeBretagne, interviennent dans les quatre domaines de recherche mais avec des profils différents. Ainsi, les publications impliquant au moins un organisme français concernent à 39 p. 100 la biologie. Les publications allemandes sont plutôt consacrées à la pathologie (24 p. 100 de ses publications). La Grande-Bretagne accorde la même importance à ces deux domaines. Mais elle est absente en 1990 et 1991. Les autres pays européens interviennent de façon plus ciblée thématiquement :
- l'Italie, la Belgique et la Suède interviennent essentiellement et assez régulièrement dans le secteur de la biologie depuis 1990 ;

- la Suisse concentre ses travaux sur les productions (exclusivement lait et produits laitiers). Ils concernent presque uniquement la période 1990-1994;

- la Belgique publie préférentiellement sur le thème de l'immunologie.

\section{Acteurs et leurs coopérations}

L'analyse des cosignatures des publications met en lumière les principaux acteurs et les réseaux actuels de collaboration. Seules les données des publications des quatre dernières années (19941998) ont été prises en compte afin d'inclure de façon plus exacte les collaborations en cours. Ces données correspondent à un ensemble de 1031 références.

\section{Organismes acteurs}

Au total, 278 organismes signataires de publications dont la moitié sont à fréquence 1 sont identifiés. C'est le signe d'une grande diversité voire d'une dispersion. Parmi ces derniers, 57 p. 100 publient seuls. Ce chiffre tendrait à montrer qu'une partie des recherches sur le dromadaire se fait de façon ponctuelle et isolée.

En ne retenant que les organismes qui publient en moyenne quatre articles par an (tableau III), on obtient une liste de 18 structures que l'on pourrait qualifier d'incontournables en matière de recherche sur le dromadaire.

Ce classement par organisme situe le Maroc dans le groupe des pays phares au même niveau que l'Arabie saoudite et juste derrière les deux organismes indiens Rajasthan Agricultural University et National Research Centre for Camels. Par ailleurs, parmi les pays du Nord, la France est le seul pays européen qui apparaît à ce niveau de fréquence.

L'analyse du nombre de copublications pour chaque organisme indique une tendance forte de certains d'entre eux à publier seul (l'Inde par exemple), à l'inverse d'autres pays comme le Maroc, le Soudan, le Kenya, la Mauritanie qui affichent une stratégie de collaboration avec d'autres pays, soit du Sud, soit européens.

Globalement l'étude des coopérations révèle qu'il n'existe pas de véritable réseau international pour la recherche sur le dromadaire (figure 5).

\section{Auteurs majeurs}

Un index a été réalisé sur le premier auteur de chaque publication afin d'identifier les experts sur le dromadaire, leur organisme et les pays d'affiliation (tableau IV).

\section{Recherches sur le chamelon}

La préoccupation de la recherche scientifique sur le chamelon est relativement récente (figure 6) et se répartit selon trois rubriques majeures : la biologie, l'élevage et la pathologie.

Depuis 1970, l'interrogation des bases de données internationales a permis de rassembler 185 références. Dans la plupart d'entre elles, le chamelon n'est pas le thème majeur. Il est abordé indirectement au travers des études sur le dromadaire. Il s'agit en général d'études comparatives entre le jeune animal et l'animal adulte. 
Tableau III

O rganismes acteurs (1994-1998)

\begin{tabular}{|c|c|c|c|}
\hline Pays & O rganisme & Fréq. des publications & Fréq. des copublications \\
\hline Inde & Rajasthan Agricultural University & 92 & 11 \\
\hline Inde & National Research Center for Camels & 73 & 12 \\
\hline Arabie saoudite & King Saud University & 49 & 0 \\
\hline Maroc & IAV Hassan II & 48 & 41 \\
\hline Inde & CCS Haryana Agricultural U niversity & 44 & 6 \\
\hline Arabie saoudite & King Faisal University & 40 & 12 \\
\hline Egypte & University of Cairo & 39 & 14 \\
\hline Soudan & University of Khartoum & 31 & 20 \\
\hline France & Inra & 31 & 28 \\
\hline Emirats arabes unis & United Arab Emirates University & 29 & 8 \\
\hline Emirats arabes unis & Central Veterinary Research Laboratory & 28 & 15 \\
\hline France & Cirad-emvt & 25 & 22 \\
\hline Egypte & Assiut University & 25 & 4 \\
\hline Pakistan & University of Agriculture & 24 & 9 \\
\hline Kenya & Kenya Trypanosomosis Research Institute & 19 & 10 \\
\hline Egypte & Zagazig University & 17 & 0 \\
\hline Egypte & University of Nairobi & 16 & 6 \\
\hline Mauritanie & Cnerv Nouakchott & 16 & 12 \\
\hline
\end{tabular}

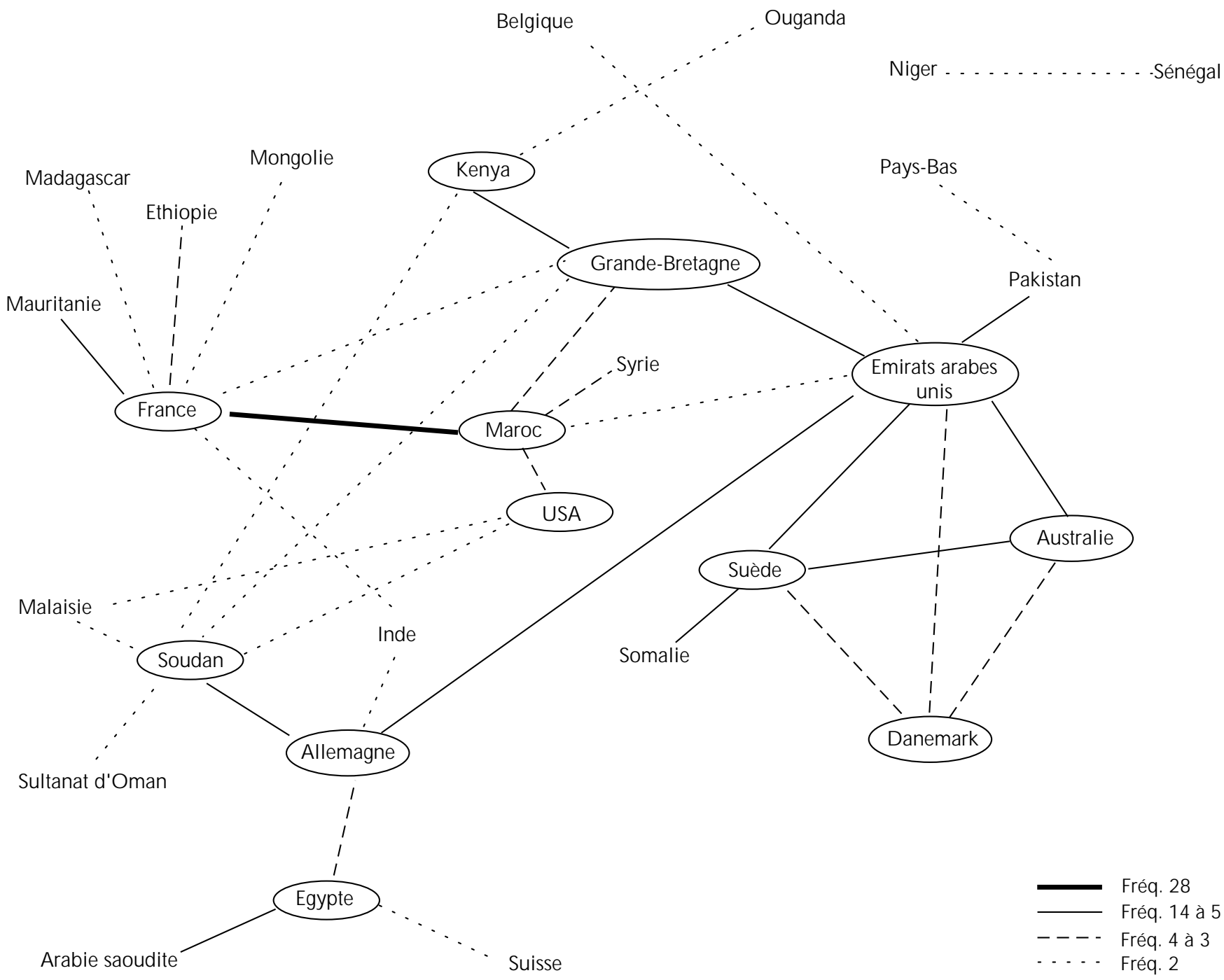

Figure 5 : réseaux des coopérations de recherche sur le dromadaire entre pays au travers des copublications (1994-1998). 


\section{Tableau IV}

Auteurs majeurs ayant publié sur le dromadaire (1994-1998)

\begin{tabular}{|c|c|c|c|}
\hline $\begin{array}{l}\text { Premier auteur } \\
\text { des publications }\end{array}$ & O rganisme d'affiliation & Pays d'affiliation & Fréq. des publications \\
\hline Gahlot T.K. & Rajasthan Agricultural U niversity & Inde & 17 \\
\hline Bengoumi M. & IAV Hassan II & Maroc & 16 \\
\hline Wernery $U$. & Central Veterinary Research Laboratory & Emirats arabes unis & 12 \\
\hline Skidmore J.A. & Camel Reproduction Center Dubai & Emirats arabes unis & 11 \\
\hline Gitao C.G. & U niversity of Nairobi & Kenya & 11 \\
\hline Fahmy L.S. & University of Cairo & Egypte & 11 \\
\hline Khalafalla A.I. & U niversity of Khartoum & Soudan & 10 \\
\hline Saber A.S. & Assiut U niversity & Egypte & 8 \\
\hline Partani A.K. & Rajasthan Agricultural U niversity & Inde & 8 \\
\hline Vyas S & $\mathrm{N}$ ational Research Center for Camels & Inde & 7 \\
\hline Patak K.M.L. & Rajasthan Agricultural University & Inde & 7 \\
\hline O ukessou M. & IAV Hassan II & Maroc & 7 \\
\hline Jain R.K. & CCS H aryana Agricultural U niversity & Inde & 7 \\
\hline Jacquiet $P$. & Cnerv Nouakchott & Mauritanie & 7 \\
\hline Faye B. & Inra puis Cirad-emvt & France & 7 \\
\hline Dia M.L. & Cnerv Nouakchott & Mauritanie & 7 \\
\hline Agarwal S.P. & N ational Research Center for Camels & Inde & 7 \\
\hline Singh A.P. & Rajasthan Agricultural U niversity & Inde & 6 \\
\hline Rai A.K. & N ational Research Center for Camels & Inde & 6 \\
\hline Olaho Mukani W. & Kenya Trypanosomiasis Research Institute & Kenya & 6 \\
\hline Nagpal A.K. & $\mathrm{N}$ ational Research Center for Camels & Inde & 6 \\
\hline Kohler Rollefson I. & Technische Hoeschule & Allemagne & 6 \\
\hline Hegazy A.A. & U niversity of Cairo & Egypte & 6 \\
\hline Duhaiman A.S. & King Saud U niversity & Arabie saoudite & 6 \\
\hline Al Jafari A.A. & King Saud University & Arabie saoudite & 6 \\
\hline Agab H. & Gedaref Veterinary Research Laboratory & Soudan & 6 \\
\hline
\end{tabular}

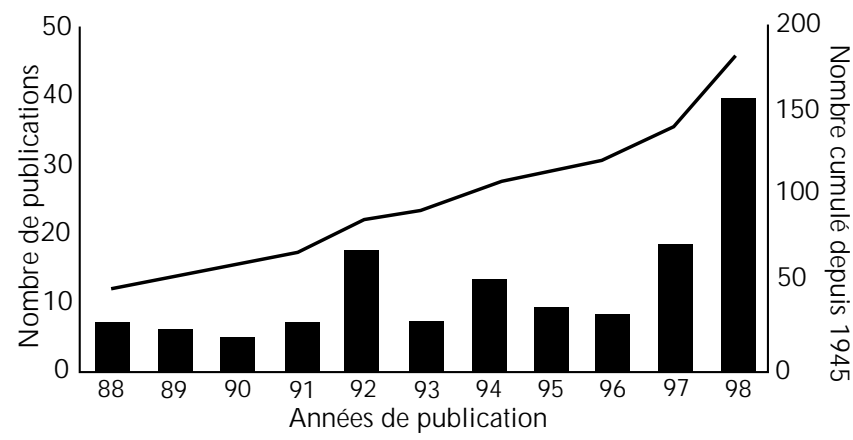

Nombre de publications

Nombre cumulé depuis 1945

Figure 6 : répartition annuelle et évolution des publications sur le chamel on.

Les travaux sur le chamelon peuvent être classés selon quatre thèmes issus du plan de classement du $\mathrm{Cd}$-rom Le Dromadaire (3) :

- les maladies ; le nombre de références sur les maladies est quantitativement le plus important (44 p. 100 des références). Les maladies infectieuses sont décrites dans la plupart des cas en comparant l'évolution de la maladie chez le jeune et chez l'adulte. Les troubles digestifs, maladies de carence, intoxications, diarrhées sont aussi abordés fréquemment ;
- la biologie/physiologie, qui comprend les études sur la morphologie, l'anatomie de l'animal ainsi que les travaux relevant de disciplines telles que la biochimie, l'endocrinologie et l'immunologie, recouvre 26 p. 100 des publications consacrées au chamelon ;

- l'élevage (28 p. 100 des références) qui concerne plus particulièrement les systèmes d'élevage, la reproduction et les performances de reproduction, les problèmes de croissance ainsi que les travaux sur la lactation, l'allaitement, la composition du lait. L'alimentation est une rubrique importante, elle est suivie par le signalement des travaux spécifiques sur le comportement et la mortalité ;

- la production qui ne comporte qu'un petit nombre de publications (2 p. 100).

\section{Supports de publication des résultats de la recherche sur le dromadaire}

Une étude des périodiques dans lesquels sont publiés les articles sur le dromadaire place en première position le Journal of Camel Practice and Research, édité par la Rajasthan Agricultural University avec 99 publications depuis seulement 1994, date de la création de cette revue. Il s'agit, de fait, de la seule revue spécialisée sur cette espèce. 
La mise en relation des thèmes de recherche avec les supports de publication montre que les thématiques maladies et biologie sont prédominantes dans les articles de périodiques alors que les communications à des congrès traitent essentiellement d'élevage et de production.

\section{CONCLUSION}

L'intérêt des scientifiques pour l'étude des grands camélidés n'est pas démenti. Au regard du nombre d'articles et de congrès, la tendance est vers une lente augmentation de la production scientifique. Toutefois, les travaux restent quantitativement marginaux en comparaison aux autres espèces d'herbivores domestiques. Cette marginalité est liée, bien sûr, à la faiblesse des effectifs mondiaux de camélidés (officiellement 20 millions, mais davantage vraisemblablement) comparés, par exemple, à la population bovine mondiale (1,3 milliard), mais aussi à la restriction géographique de l'aire de distribution, le dromadaire étant un animal fortement associé à des pays arides et semi-arides où, dans la plupart des cas, la recherche zootechnique et vétérinaire ne répond pas aux besoins

\section{Summary}

Faye B., Bonnet P., Charbonnier G., Marti A. Assessing camel research by a bibliometrical study of publications with an emphasis on the camel calf

Camel research as well as the main collaborations between players in the field are analyzed based on a bibliometrical study of the last ten years (journal publications, conference proceedings). A special consideration is given to camel calf research

Key words: Camel - Young animal - Documentation Research - Cooperation - Developed country - Developing country. réels des pays concernés. Il faut donc voir dans l'analyse présentée ici, à la fois un motif d'espoir de voir le développement d'une recherche caméline effective par une coopération Nord-Sud et Sud-Sud soutenue, et une certaine modestie : les 7100 références répertoriées depuis 1779 (!) dans la base de données analysée, n'équivalent guère plus qu'au même nombre de références consacrées à la vache depuis moins de deux ans.

\section{BIBLIO GRAPHIE}

1. BONNET P. éd., 1998. Actes de l'atelier Dromadaires et chameaux, animaux laitiers, Nouakchott, Mauritanie, 24-26 octobre 1994. Montpellier, France, Cirad-emvt, $301 \mathrm{p}$.

2. FAYE B. éd., 1997. Le guide de l'élevage du dromadaire. Libourne, France, Sanofi, $126 \mathrm{p}$

3. FAYE B., MEYER C., MARTI A., 1999. Le dromadaire. Cd-rom. Montpellier, France, Cirad.

4. SAINT-MARTIN G. éd., 1993. Actes de l'atelier Peut-on améliorer les performances de reproduction des camelins, Paris, France, 10-12 septembre 1990. Maisons-Alfort, France, Cirad-emvt, 437 p.

\section{Resumen}

Faye B., Bonnet P., Charbonnier G., Marti A. Estado de las investigaciones sobre el dromedario a partir del análisis bibliográfico de las publicaciones - caso particular de las investigaciones sobre el camello joven

Se analizan las actividades de investigación sobre el dromedario, así como las principales colaboraciones entre los actores de este sector, apoyándose sobre un estudio bibliográfico, comprendiendo los últimos 10 años (publicaciones en las revistas periódicas y los congresos). Se da particular atención a las investigaciones sobre el camello joven.

Palabras clave: Dromadario - Animal joven - Documentación Investigación - Cooperación - Pais desarollado - Pais en desarollo. 